\title{
BMJ Open Qingdao Port Cardiovascular Health Study: a prospective cohort study
}

\author{
Erica S Spatz, ${ }^{1,2}$ Xianyan Jiang, ${ }^{3}$ Jiapeng Lu, ${ }^{4}$ Frederick A Masoudi, ${ }^{5}$ \\ John A Spertus, ${ }^{6}$ Yongfei Wang, ${ }^{1,2} \mathrm{Xi} \mathrm{Li},{ }^{4}$ Nicholas S Downing, ${ }^{1}$ Khurram Nasir, ${ }^{8,9}$ \\ Xue Du, ${ }^{4}$ Jing Li, ${ }^{4}$ Harlan M Krumholz, ${ }^{1,2,10,11}$ Xiancheng Liu, ${ }^{3,7}$ Lixin Jiang ${ }^{4}$
}

To cite: Spatz ES, Jiang $X$, Lu J, et al. Qingdao Port Cardiovascular Health Study: a prospective cohort study. BMJ Open 2015;5:e008403. doi:10.1136/bmjopen-2015008403

- Prepublication history and additional material is available. To view please visit the journal (http://dx.doi.org/ 10.1136/bmjopen-2015008403)

ESP, XJ and JLu contributed equally to this work. HMK XLiu and LJ are co-senior authors.

Received 4 April 2015 Revised 24 October 2015 Accepted 2 November 2015

CrossMark

For numbered affiliations see end of article.

Correspondence to Professor Lixin Jiang; jiang|@fwoxford.org

\section{ABSTRACT}

Purpose: In China, efforts are underway to respond to rapidly increasing rates of heart disease and stroke. Yet the epidemiology of cardiovascular disease in China may be different from that of other populations. Thus, there is a critical need for population-based studies that provide insight into the risk factors, incidence and outcomes of cardiovascular disease in China. The Qingdao Port Cardiovascular Health Study is designed to investigate the burden of cardiovascular disease and the sociodemographic, biological, environmental and clinical risk factors associated with disease onset and outcomes.

Participants: For this study, from 2000 through 2013, 32404 employees aged 18 years or older were recruited from the Qingdao Port Group in China, contributing 221923 annual health assessments. The mean age at recruitment was $43.4(\mathrm{SD}=12.9) ; 79 \%$ were male. In this ongoing study, annual health assessments, governed by extensive quality control mechanisms, include a questionnaire (capturing demographic and employment information, medical history, medication use, health behaviours and health outcomes), physical examination, ECG, and blood and urine analysis. Additional non-annual assessments include an X-ray, echocardiogram and carotid ultrasound; bio-samples will be collected for future genetic and proteomic analyses. Cardiovascular outcomes are accessed via self-report and are actively being verified with medical insurance claims; efforts are underway to adjudicate outcomes with hospital medical records.

Findings to date: Early findings reveal a significant increase in cardiovascular risk factors from 2000 to 2010 (hypertension: $26.4-39.4 \%$; diabetes: $3.3-8.9 \%$; hyperlipidaemia: $5.0-33.6 \%$; body mass index $>28$ m/kg ${ }^{2}: 14.1-18.6 \%$ ).

Future Plans: We aim to generate novel insights about the epidemiology and outcomes of cardiovascular disease in China, with specific emphasis on the potentially unique risk factor profiles of this Chinese population. Knowledge generated will be disseminated in the peerreviewed literature, and will inform populationbased strategies to improve cardiovascular health in China.

Trial registration number: NCT02329886.

\section{Strengths and limitations of this study}

- This is a prospective cohort study conducted for over 10 years.

- The study collected a variety of information including cardiovascular risk factors, clinical outcomes and bio-samples, allowing a wide ranging assessment of risk factors and genetic factors related to cardiovascular disease onset and cardiovascular outcomes.

- Findings will have broad implications for China's workforce and for employer-based healthcare.

- Participants are limited to a single company and may not be representative of the larger population of China.

\section{INTRODUCTION}

Epidemiological studies are the cornerstone for understanding cardiovascular risk factors and disease. Decades of research extending from the Framingham Heart Study and other cardiovascular studies (eg, Atherosclerosis Risk in Communities Study) provide insight into the complex interplay between biology, behaviour, environment and genes, on the risk of cardiovascular disease (CVD) and stroke. ${ }^{1-4}$ However, existing studies of the epidemiology of CVD may have limited relevance to other populations, particularly in countries with different health behaviours and environments.

The relationships between risk factors and CVD outcomes differ between populations. As demonstrated in the WHO Multinational Monitoring of Trends and Determinants in Cardiovascular Disease (MONICA) Project, along with other studies, metabolic, dietary and other lifestyle factors do not consistently portend the same cardiovascular risk across populations. $^{5-11}$ The graded relationship between higher cholesterol levels and mortality from coronary heart disease varies among Western countries ${ }^{12}$ and may differ in China. $^{13} 14$ Studies also demonstrate 
differences in the epidemiology of stroke, with the slope of the relationship between blood pressure and stroke steeper among Asian populations as compared with Australian populations, ${ }^{15}$ and haemorrhagic stroke occurring more frequently in Chinese populations than in Western populations. ${ }^{16}$ In summary, the epidemiology of heart disease and stroke in China, and its associated risk factors, may be different from that of other populations, and thus may have different implications for intervention and prognosis. Understanding potential differences in the epidemiology and outcomes of CVD in China requires population-based studies.

Well-conducted, population-based prospective studies focused on CVD are critical, especially as China is experiencing remarkable changes in the cardiovascular risk of its population. ${ }^{17-20}$ Similar to other developing countries undergoing an epidemiological transition, there has been a dramatic rise in the prevalence of noncommunicable diseases such as CVD. ${ }^{13}{ }^{21}$ Approximately 1 in 5 Chinese adults aged 18 years or older (approximately 230 million people) have CVD, the prevalence of which is expected to double in the next decade. ${ }^{22} 23$ Beyond the expected rise in CVD associated with longer life expectancy, ${ }^{24}$ other factors such as urbanisation, ${ }^{25}$ environmental pollutants ${ }^{26-28}$ and sociocultural shifts, ${ }^{29}$ have been associated with the increasing prevalence of obesity, ${ }^{30}$ hypertension, ${ }^{31}$ diabetes ${ }^{32}$ and smoking, ${ }^{33}$ though their relationship with CVD in China has not been well described. ${ }^{34}{ }^{35}$ To date, there are few contemporary, prospective, population-based studies to identify factors associated with heart disease and stroke in the Chinese population. ${ }^{36} 37$

Accordingly, the Qingdao Port Group and its affiliated hospital, Qingdao Fuwai Hospital, established the
Qingdao Port Cardiovascular Health Study in 2000, a prospective cohort study of employees of the Group, with the aims of monitoring the incidence of CVD and associated risk factors, and identifying risk factors associated with the incidence of CVD and cardiovascular outcomes. Knowledge gained from this study will be used to guide China's efforts in preventing and managing heart disease and stroke. In this paper, we describe the design, cohort and investigational priorities of the Qingdao Port Cardiovascular Health Study.

\section{COHORT DESCRIPTION}

\section{Study design and investigational priorities}

The Qingdao Port Cardiovascular Health Study was started in 2000. The study is designed to monitor CVD trends and to understand risk factors contributing to chronic conditions with a focus on heart disease and stroke. ${ }^{38}{ }^{39}$ Specifically, the main investigational priorities are: (1) surveillance of cardiovascular risk factors, disease and outcomes; (2) investigation of sociocultural, biological, behavioural, social and environmental factors on work productivity, CVD onset and CVD health outcomes; (3) development and validation of risk models to predict cardiovascular events; and (4) genetic and proteomic analysis of biological samples to elucidate disease mechanisms and inherited risk profiles.

\section{Setting, recruitment and eligibility}

The Qingdao Port Group of the Shandong province in China is one of the oldest and largest shipping and trade companies in the world (figure 1). The workforce is diverse, with administrative personnel, technical personnel (eg, pilots, computer analysts and information technology

Figure 1 Examination sites for the Qingdao Port Cardiovascular Health Study.

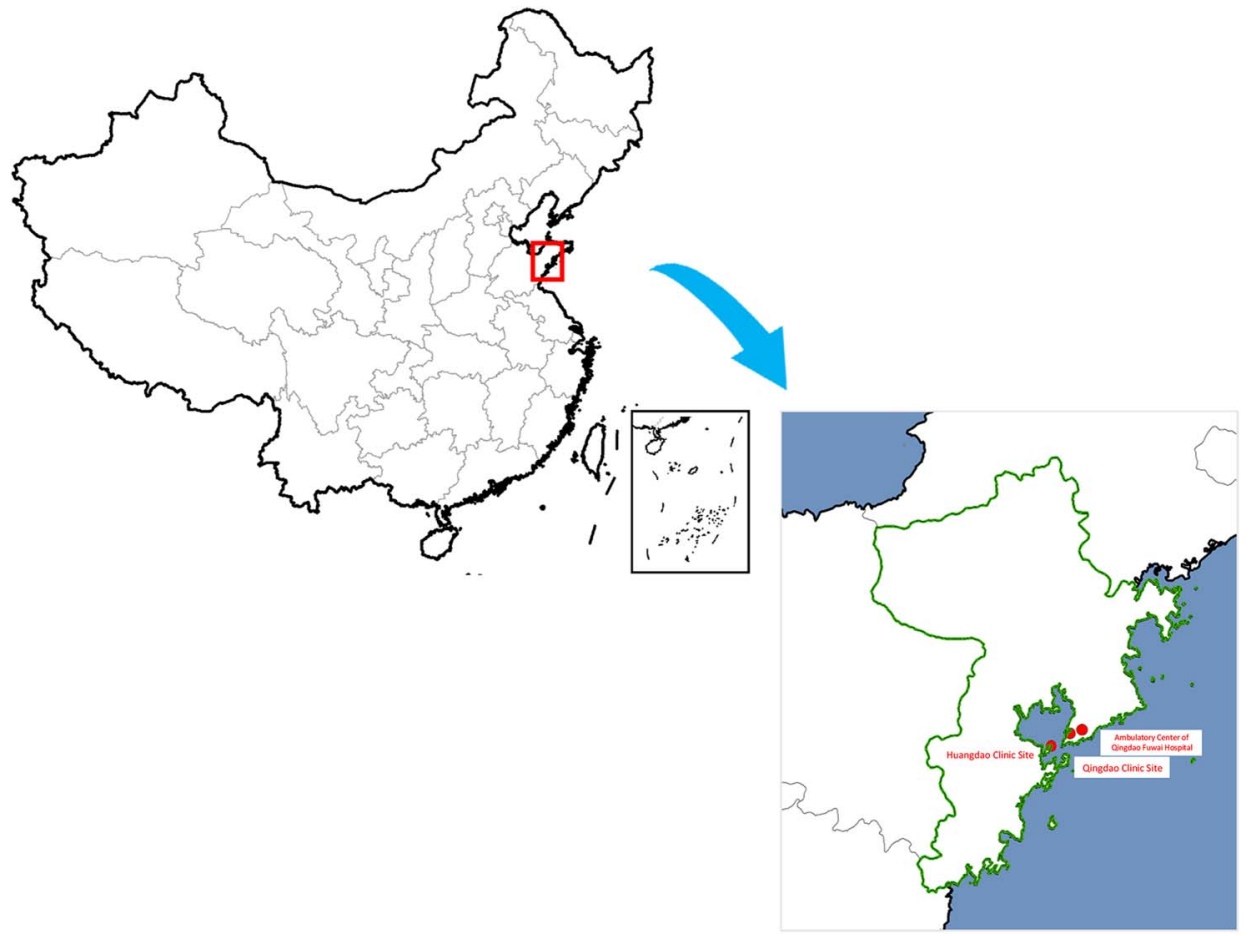


specialists, police, teachers, physicians, nurses and other hospitals staff), production operators, ship workers, dockworkers and drivers. Among them, ship workers and dockworkers account for the majority of all staff.

The company contributes to each employee's health insurance plan, which is administered by the Qingdao Medical Insurance Bureau. The plan covers annual health assessments, on-site ambulatory medical care and coverage of inpatient services rendered at the Qingdao Fuwai Hospital-the local hospital and partner in this study - as well as other public hospitals in Qingdao city. All employees aged 18 years or older, who present for an annual health assessment, are eligible for study participation. The only exclusion is workers who are migrant, as they are not covered by the local health insurance and do not receive annual health examinations, effectively rejecting them from study participation.

Prior to 2013, employees were verbally asked to participate. Since then, however, employees sign an informed consent document prior to enrolment (see online supplementary appendix 1: translated informed consent document). Among the employees who present for a health assessment, almost all $(98 \%)$ agreed to participate in the study. At each annual visit, employees renew their agreement to participate. Participants are informed that the data are confidential.

The annual health assessments, offered at the three work sites, serve as the platform for data collection. The organisational structure for the study is presented in figure 2.

\section{Health assessment components}

Annual health assessments, which are performed by 35 trained physicians and nurses, consist of a questionnaire, physical examination, ECG, laboratory testing and bio-sampling. In 2013, echocardiography and carotid ultrasound were additionally performed, and biosamples were collected for future analysis. Currently, there are 466 unique variables summarised by domain in online supplementary appendix 2.

\section{Questionnaire}

A standardised questionnaire is administered by trained personnel during a face-to-face annual health assessment (see online supplementary appendix 3). Questions are adapted from prior epidemiological studies ${ }^{40}$ and, when available, questionnaires validated in Chinese are used. ${ }^{41}$ The following domains are assessed: demographics (eg, income, education, family structure); personal medical history (eg, medical history, medication use and adherence); family history; health behaviours (eg, nutritional intake; physical activity; ${ }^{42}$ smoking; alcohol use/ abuse); healthcare utilisation (eg, hospitalisations; use of traditional Chinese medicine); menopause (including hormone use); emotional health (eg, anxiety, stress); and employment structure (eg, physical labour; shift-work).

\section{Physical examination}

A detailed, comprehensive physical examination is conducted at baseline by trained medical staff. Nurses measure height, weight, waist circumference, hip circumference, blood pressure and heart rate. Physicians perform a detailed eye and ear examination, dental assessment, thyroid palpation, cardiopulmonary auscultation and abdominal examination. Nurses and physicians follow standardised protocols for measurement and examination. Height $(\mathrm{cm})$, weight $(\mathrm{kg})$, and waste and hip circumferences are measured using sonographic
Figure 2 Organisational structure of the Qingdao Port Cardiovascular Health Study.

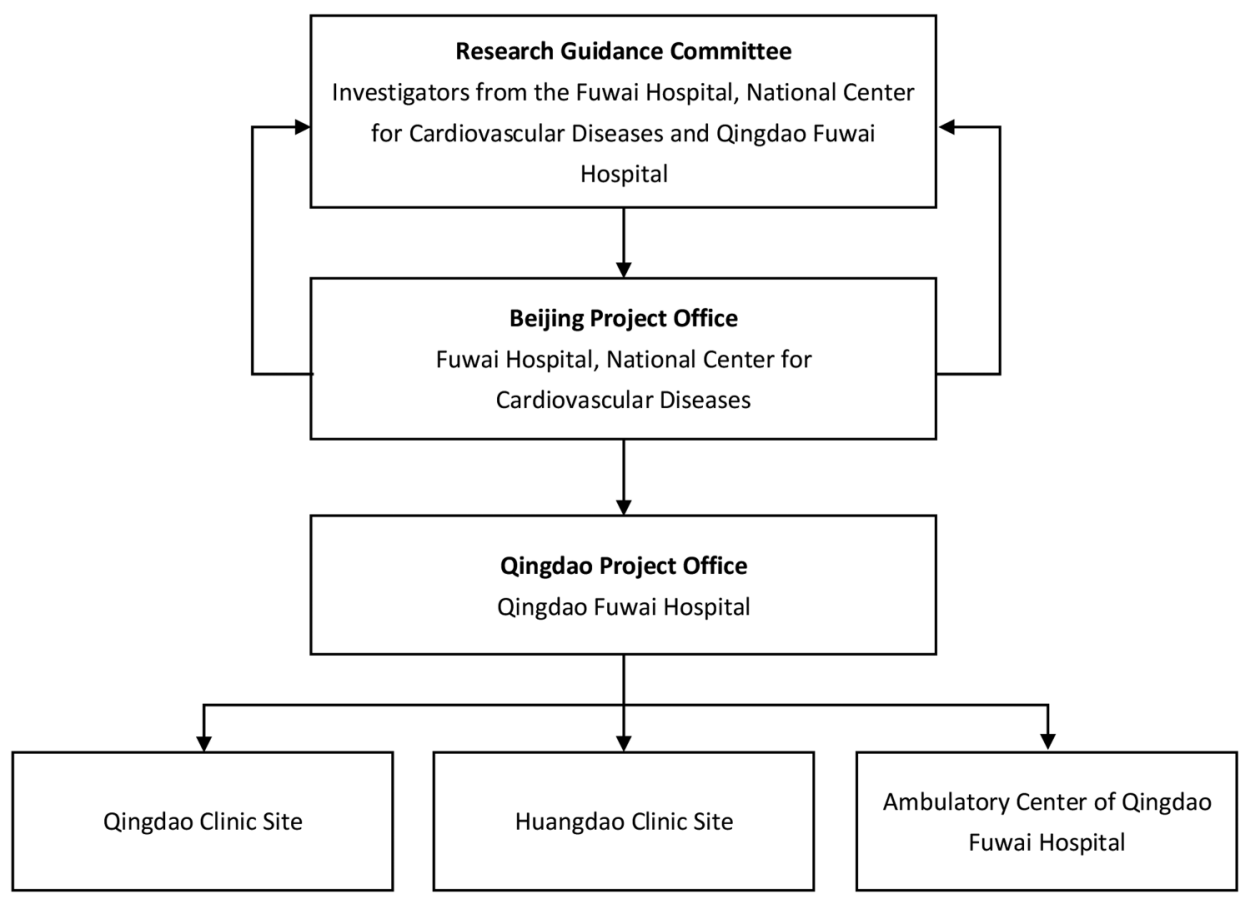


technology (Ultrasound Height \& Weight Machine, TCS-160D-W/H, Shenzhen Sonka Electronic Technology Co, Ltd, Guangdong, China), averaged to the nearest 0.1 unit. Instruments are calibrated weekly with phantom mannequins. Blood pressure and heart rate are measured after the patient has been seated for 5 min, using a calibrated electronic sphygmomanometer. Blood pressure measurement is performed by placing the cuff on the dominant arm, at the level of the heart, and taking the average of three measurements spaced $1 \mathrm{~min}$ apart. Heart rate is recorded contemporaneously with each blood pressure measurement.

\section{Laboratory tests}

Venipuncture, performed by trained nurses, is obtained annually. Some blood chemistries are repeated each year (eg, fasting glucose, lipid profile, creatinine). Tests performed intermittently include liver and thyroid function.

\section{Bio-samples for future analysis}

Since 2013, participants have been invited to submit biosamples, including blood, urine and saliva samples, for storage, to be used for future DNA, RNA and biochemical analysis. Over $90 \%$ of study participants have consented to date. Whole blood samples are drawn into EDTA, serum gel, heparin lithium and Tempus blood RNA tubes (Applied Biosystems, Foster City, California, USA) for future gene expression analysis. When stored in Tempus tubes at $-80^{\circ} \mathrm{C}$, the whole blood RNA retains high integrity and purity for over a 6 -year period. ${ }^{43}$ To collect saliva, participants are asked to chew a cotton roll for $45 \mathrm{~s}$; the cotton roll is placed into a Salivette tube (Sarstedt AG \& Co, Nümbrecht, Germany). Within 24 h, trained nurses centrifuge blood and saliva samples, and divide and transfer the samples into cryovials, according to standard protocol. Urine is transferred directly into cryovials. All samples are immediately stored at $-80^{\circ} \mathrm{C}$, and are transported to Fuwai Hospital, National Center for Cardiovascular Diseases (NCCD), for long-term storage, within 2 months.

\section{Additional testing}

ECG is performed every year. Additionally, specific cardiovascular screening tests were conducted during different years including: chest X-ray, echocardiogram and carotid ultrasound. All imaging tests are conducted by certified technologists in accordance with standards set forth by the Ministry of Health.

\section{Follow-up}

Follow-up health assessments are performed annually. While the employee population is dynamic, among the 32404 study participants from 2000 to 2013, more than half $(18111 ; 55.9 \%)$ have 5 or more years of follow-up; $11537(35.6 \%)$ participants have 10 or more years of follow-up (figure 3). Of the original cohort of 11201 people who started in 2000, 7433 were not participating in annual follow-up as of 2013. For example, among 22128 study participants who enrolled between 2000 and 2010, 3280 (14.8\%) had no follow-up visit after 2011 and before the end of the 2013 measurement period. These employees may have left the company or declined further participation; it is also possible, though less likely, that they missed three consecutive visits and will return in 2014. Distinguishing individuals with interrupted visits from permanent drop-outs is a goal for the future (table 1).

The components of the follow-up visit are the same as the baseline assessment and include a face-to-face interview, physical examination, laboratory testing and imaging examinations specified for that year. New participants are recruited each year (figure 4) and, since

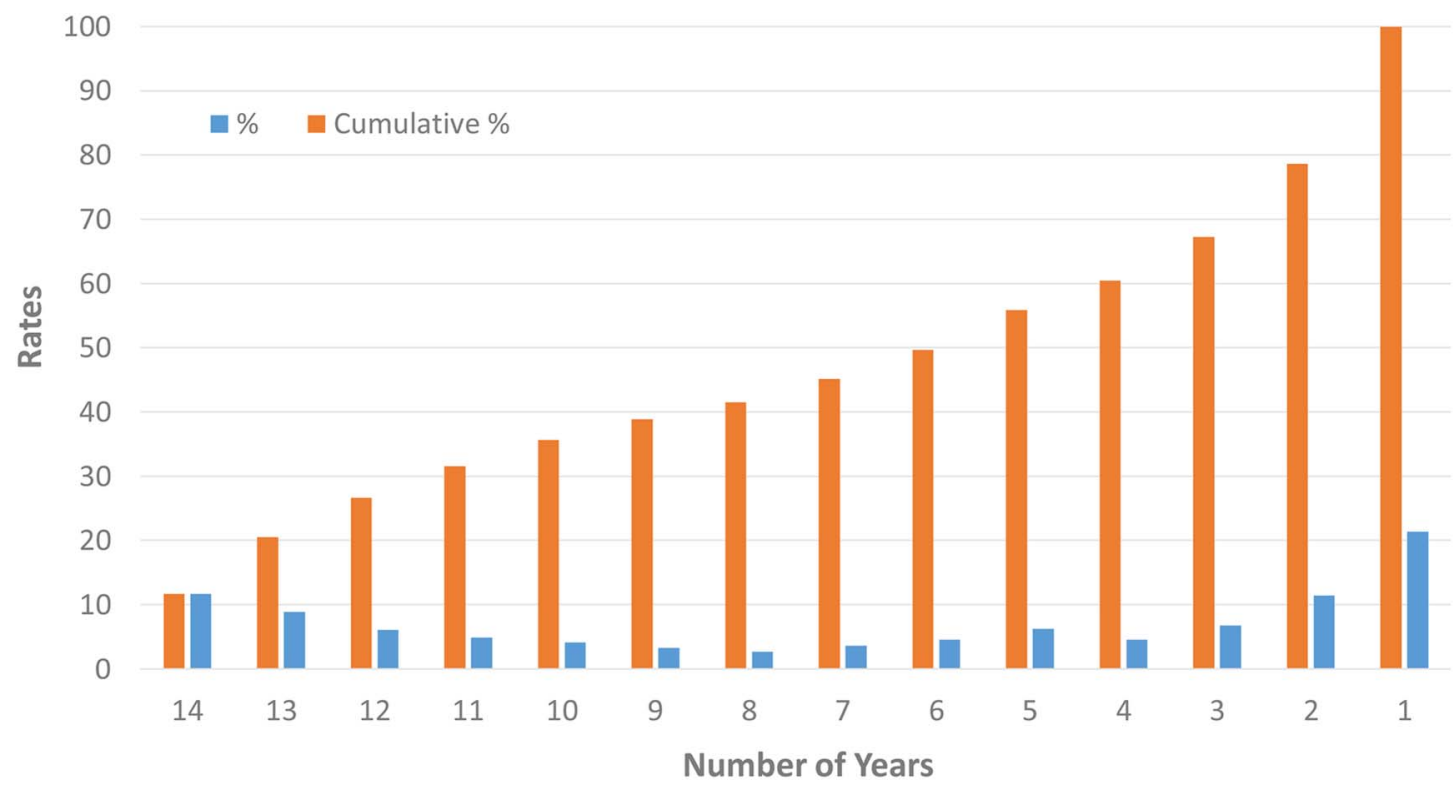

Figure 3 Distribution of frequency of study participant follow-up. 
Table1 Follow-up among study participants

\begin{tabular}{|c|c|c|c|c|}
\hline \multirow[b]{2}{*}{ Description } & \multirow[b]{2}{*}{ Total cohort } & \multirow[b]{2}{*}{ Returning participants } & \multicolumn{2}{|c|}{ Non-returning participants } \\
\hline & & & $\mathbf{N}$ & Percent \\
\hline 2000 to $2001-2013$ & 11201 & 11201 & 0 & 0.00 \\
\hline $2000-2001$ to $2002-2013$ & 12363 & 12260 & 103 & 0.83 \\
\hline 2000-2002 to 2003-2013 & 13474 & 13291 & 183 & 1.36 \\
\hline 2000-2003 to 2004-2013 & 15849 & 15212 & 637 & 4.02 \\
\hline $2000-2005$ to $2006-2013$ & 16614 & 15637 & 977 & 5.88 \\
\hline 2000-2006 to 2007-2013 & 17136 & 15838 & 1298 & 7.57 \\
\hline $2000-2007$ to $2008-2013$ & 17963 & 16263 & 1700 & 9.46 \\
\hline 2000-2008 to 2009-2013 & 18947 & 16833 & 2114 & 11.16 \\
\hline 2000-2009 to 2010-2013 & 20221 & 17476 & 2745 & 13.57 \\
\hline 2000-2010 to $2011-2013$ & 22128 & 18848 & 3280 & 14.82 \\
\hline $2000-2011$ to $2012-2013$ & 22670 & 18775 & 3895 & 17.18 \\
\hline $2000-2012$ to 2013 & 24636 & 18521 & 6115 & 24.82 \\
\hline
\end{tabular}

2003, participants have or are being followed into retirement, up until death. In China, the statutory retirement age for general workers is 60 years for men and 5055 years for women, depending on their job position. Among heavy labourers, the retirement age is 55 years for men and 45 years for women. Senior professionals may work until the age of $65-70$ years. Since 2003, retirees account for approximately one-quarter of all annual health assessments each year, with approximately 7000 retirees in the study.

\section{Outcomes}

The following cardiovascular outcomes are collected: unstable angina; acute myocardial infarction; revascularisation with either percutaneous coronary intervention or coronary artery bypass grafting; heart failure; stroke; and all-cause death. As part of the annual health questionnaire, participants are asked to report information on newly diagnosed diseases, hospitalisations and treatments that occurred in the previous year. Self-reported health outcomes are actively being linked with medical claims data; since all employees have the same insurance plan, complete insurance claims data are available for all years except 2000 and 2001, years in which not all employees were insured with the same plan. Additionally, since 2013, medical claims have been linked with health assessment data every 3 months, independent of whether participants self-reported a clinical outcome. From the medical claims, the following information is abstracted: admission date, discharge date, discharge diagnoses and corresponding International Classification of Disease (ICD) codes; and discharge status. Deaths are monitored by the insurance plan and shared with the Qingdao Port Company and study

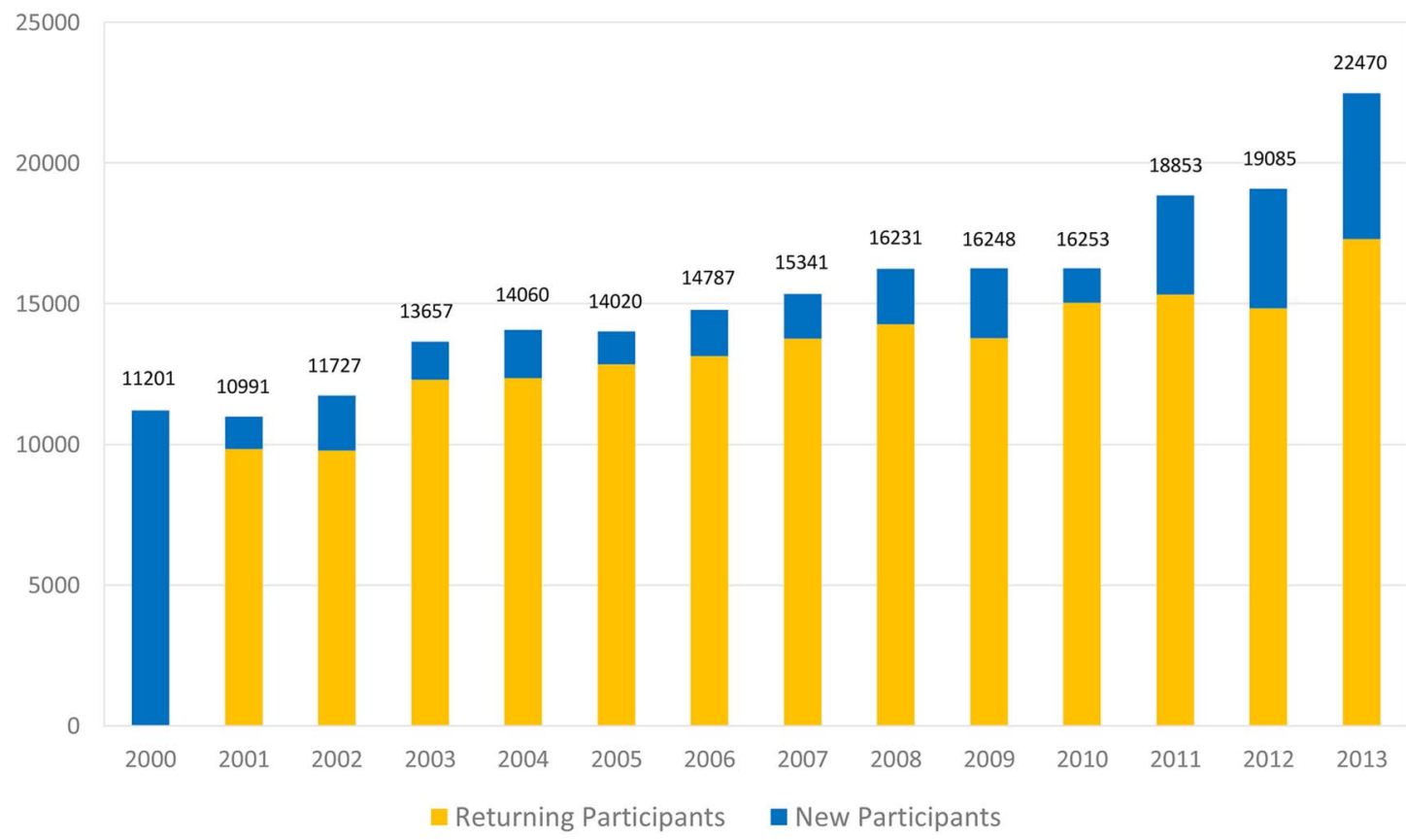

Figure 4 Distribution of new and returning study participants per year. 
group, which prompts the collection of death certificates and autopsy reports to elucidate the cause of death. Hospital medical records are also assessed to verify cause of death. If hospitalisation records or other supportive records are not available, interviews with the person's relatives and physicians are conducted to acquire the date and cause of death.

Efforts are underway to adjudicate a subset of outcomes using hospital medical records. In 2013, 855 participants reported hospitalisations, 300 of which were reported to have occurred at Qingdao Fuwai Hospital, all of which were verified. Among patients who reported being hospitalised outside of Qingdao Fuwai Hospital $(n=555)$, medical records from 246 participants were obtained. These records came directly from participants, though there are efforts to develop a process that will enable the direct collection of study participants' medical records from outside hospitals. Consistent with other cardiovascular research protocols, ${ }^{44}$ adjudicators will review the clinical diagnosis and the claims-based ICD code, and compare them with self-reported data. Discrepancies between the medical record and claims data are resolved by the research guidance committee in Fuwai Hospital, NCCD.

\section{Quality assurance and control}

All research staff participate in 2 days of intensive training focused on administering the questionnaire and learning the protocols for blood pressure measurements, anthropometry, blood collection and sample processing, ECG and data entry. Further operational training is conducted at the study sites. Each year, all equipment, including the biochemical analyser, ultrasound machine, X-ray machine, electronic sphygmomanometer and ultrasound stadiometers are tested and calibrated by the Qingdao Institute of Measurement and Testing and the Qingdao Institute of Weighing Apparatus Management; annual calibration certificates by these two institutes are required. The validity of all measurements is checked monthly by examination of data outliers and missing fields. Additionally, site monitoring is conducted by two trained research staff members from Fuwai Hospital, NCCD, every 3-6 months, assessing: (1) the completeness of the documentation and (2) fidelity to protocol for recruitment, health screening, physical examination, and sample collection and processing. Finally, annual meetings are held to discuss problems with measurement and data entry, and to optimise study processes.

To improve the validity and reliability of the measurement of cardiovascular risk factors, questions were adapted from prior epidemiological studies in China, such as questions assessing smoking and dietary patterns. ${ }^{40}$ In 2013, questionnaires that were previously validated in Chinese were incorporated. We used the Alcohol Use Disorders Identification Test (AUDIT) questionnaire to identify persons with hazardous and harmful patterns of alcohol consumption, ${ }^{41}$ the International Physical Activity Questionnaire (IPAQ) to assess physical activity ${ }^{42}$ and the EuroQoL (EQ-5D) to measure health status. ${ }^{45}$

The definition of hyperlipidaemia changed from earlier years in accordance with the level of data available. For example, in some prior years, no blood lipid tests were performed; in these years, only self-report and medication use were used to define hyperlipidaemia. Since 2010, hyperlipidaemia has been defined as: either self-report of high cholesterol or dyslipidaemia (either: total cholesterol $\geq 5.2 \mathrm{mmol} / \mathrm{L}$ or low-density lipoprotein $\geq 3.4 \mathrm{mmol} / \mathrm{L}$ or high-density lipoprotein $<1.0 \mathrm{mmol} / \mathrm{L}$ or triglycerides $\geq 1.7 \mathrm{mmol} / \mathrm{L}$ ) or use of a lipid-lowering medication.

From 2000 to 2012, data were collected on paper and double-entered by two different researchers into a computer-based system, to reduce error. Since 2013, results from the questionnaire, physical examination, laboratory testing and other examinations have been collected electronically with an internet-based data management system developed specifically for the study. To increase the validity and reliability of the data, this system includes predefined data formats, predetermined data ranges for quantitative data, and required fields. After research personnel enter the data, internal data checks are performed to confirm that the data are correctly entered. Finally, prior to the beginning of each measurement year, all personal information is verified (name, sex, ID number, work ID, company, department, contact telephone number and address); these steps improve the accuracy of participants' information, decrease the duration of face-to-face interview time and facilitate linkage with medical insurance records.

\section{Data security and management}

All data are treated as protected health information, and are securely stored in an encrypted and passwordprotected database. Laptops are password protected, and all research personnel have individual passwords to login to the system. All data between the three sites are independent, which means research personnel only have access to the data collected at their own site.

All source data, including questionnaires, health assessment results, hospitalisation and clinical medical records, are securely stored in the three clinic sites of the Qingdao Fuwai Hospital. The electronic version of the files as well as the image files of ECG and chest X-rays are securely stored on the servers of Qingdao Fuwai Hospital. The Research Guidance Committee, comprised of investigators from the Fuwai Hospital, NCCD, and Qingdao Fuwai Hospital, make the ultimate decisions on the usage of the data, governed by institutional review board approval.

\section{Cohort}

From 2000 through 2013, a total of 32404 study participants contributed 221923 health assessments. In this cohort, the mean age was $43.4(\mathrm{SD}=12.9) ; 79 \%$ were male, consistent with the demographics of the company. 
Participants ranged in occupation: loading/unloading (9.7\%); warehouse clerk (8.0\%); ship crew (18.8\%); other shipping and dockworkers (29.9\%); management $(19.9 \%)$; other $(13.7 \%)$.

\section{Findings to date}

Table 2 compares cardiovascular risk factors across three time periods (2000 to 2005 to 2010), demonstrating an increased prevalence of hypertension $(26.4 \%$ to $38.6 \%$ to $39.4 \%$ ) and diabetes (3.3\% to $5.9 \%$ to $8.9 \%$ ), respectively. The 2005 estimates for hypertension and diabetes are similar to those observed in the 2005 International Collaborative Study of CVD in Asia (InterAsia). ${ }^{46}$ Rates of hyperlipidaemia increased from $5 \%$ to $12.4 \%$ to $33.6 \%$ across study years; however, full blood lipid panels were not conducted between 2001 and 2006. These rates are lower than those observed in a meta-analysis in which $41 \%$ of community-residing adults had dyslipidaemia. ${ }^{47}$

\section{Strengths and limitations}

The Qingdao Port Cardiovascular Health Study is uniquely positioned to measure disease incidence and trends, characterise the complex relationships between biology, environment, culture and behaviour with disease onset, trajectories and outcomes, and ultimately

Table 2 Trends in characteristics of study participants (2000-2010)

\begin{tabular}{|c|c|c|c|c|c|c|}
\hline \multirow[b]{2}{*}{ Description } & \multicolumn{2}{|l|}{2000} & \multicolumn{2}{|l|}{2005} & \multicolumn{2}{|l|}{2010} \\
\hline & $\mathbf{n}$ & Percent & $\mathbf{n}$ & Percent & $\mathbf{n}$ & Percent \\
\hline Total participants & 12023 & & 14152 & & 16378 & \\
\hline Retirees & NA & NA & 3369 & 23.8 & 4594 & 28.1 \\
\hline \multicolumn{7}{|l|}{ Demographics } \\
\hline Age (mean; SD) & 39 & 8.7 & 45 & 12.0 & 45 & 13.7 \\
\hline \multicolumn{7}{|l|}{ Gender } \\
\hline Male & 9227 & 76.7 & 10945 & 77.3 & 13076 & 79.8 \\
\hline Female & 2796 & 23.3 & 3207 & 22.7 & 3302 & 20.2 \\
\hline \multicolumn{7}{|l|}{ Cardiovascular profile } \\
\hline \multicolumn{7}{|l|}{ High blood pressure* } \\
\hline Yes & 3176 & 26.4 & 5457 & 38.6 & 6451 & 39.4 \\
\hline Missing & 0 & 0 & 4 & $<0.1$ & 4 & $<0.1$ \\
\hline \multicolumn{7}{|l|}{ Diabetes† } \\
\hline Yes & 396 & 3.3 & 838 & 5.9 & 1458 & 8.9 \\
\hline Missing & 0 & 0 & 0 & 0 & 4 & $<0.1$ \\
\hline \multicolumn{7}{|l|}{ Hyperlipidaemiał } \\
\hline Yes & 600 & 5.0 & 1749 & 12.4 & 5499 & $33.6 \ddagger$ \\
\hline Missing & 0 & 0 & 565 & 4.0 & 4 & $<0.1$ \\
\hline \multicolumn{7}{|l|}{ Acute myocardial infarction } \\
\hline \multirow[t]{2}{*}{ Yes } & NA & NA & 66 & 0.5 & 128 & 0.8 \\
\hline & & & 50 & 0.4 & 301 & 1.8 \\
\hline \multicolumn{7}{|l|}{ Stroke } \\
\hline Yes & 66 & 0.5 & 130 & 0.9 & 188 & 1.1 \\
\hline Missing & 3 & $<0.1$ & 49 & 0.4 & 320 & 2.0 \\
\hline \multicolumn{7}{|l|}{ Anthropometrics } \\
\hline \multicolumn{7}{|l|}{ Waist $(\mathrm{cm})$} \\
\hline Mean (SD) & 81.6 & 11.2 & 83.9 & 9.6 & 86.6 & 12.3 \\
\hline Missing & 0 & 0 & 292 & 2.1 & 215 & 1.3 \\
\hline \multicolumn{7}{|l|}{$\mathrm{BMI}\left(\mathrm{cm} / \mathrm{kg}^{2}\right)$} \\
\hline Missing & 25 & 0.2 & 277 & 2.0 & 190 & 1.2 \\
\hline Normal body weight $(<24)$ & 5852 & 48.7 & 5317 & 37.6 & 6313 & 38.6 \\
\hline Overweight (24 to <28) & 4456 & 37.1 & 5972 & 42.2 & 6831 & 41.7 \\
\hline Obese $(\geq 28)$ & 1690 & 14.1 & 2586 & 18.3 & 3044 & 18.6 \\
\hline \multicolumn{7}{|l|}{ Systolic blood pressure } \\
\hline Missing & 0 & 0 & 58 & 0.4 & 63 & 0.4 \\
\hline Mean (SD) & 121 & 17.1 & 122 & 18.3 & 125 & 20.9 \\
\hline \multicolumn{7}{|l|}{ Diastolic blood pressure } \\
\hline Mean (SD) & 80 & 12.1 & 82 & 11.6 & 82 & 11.2 \\
\hline \multicolumn{7}{|c|}{$\begin{array}{l}\text { *Self-report or mean blood pressure } \geq 140 \text { systolic or } \geq 90 \mathrm{~mm} \mathrm{Hg} \text { diastolic or antihypertensive medication therapy. } \\
\text { †Self-report or fasting glucose } \geq 7.0 \mathrm{mmol} / \mathrm{L} \text { or glucose-lowering medication. } \\
\ddagger \text { } \text { Self-report or lipid-lowering therapy for year } 2000 \text {; self-report only for year } 2005 \text {; self-report or dyslipidaemia (either: total cholesterol } \\
\geq 5.2 \mathrm{mmol} / \mathrm{L} \text { or low-density lipoprotein } \geq 3.4 \mathrm{mmol} / \mathrm{L} \text { or high-density lipoprotein }<1.0 \mathrm{mmol} / \mathrm{L} \text { or triglycerides } \geq 1.7 \mathrm{mmol} / \mathrm{L} \text { ) or lipid lowering } \\
\text { medication therapy for year } 2010 \text {. } \\
\text { BMI, body mass index; NA, not available. }\end{array}$} \\
\hline
\end{tabular}


inform the approach to CVD prevention and population health, especially among China's urban, working population. Knowledge generated from the population of the Qingdao Port Cardiovascular Health Study will have broad implications for China's workforce and for employer-based healthcare, where large employers contract with health systems to provide comprehensive healthcare services to their employees, including health screenings, outpatient medical care and inpatient services. In 2006, approximately 160 million people (about $28 \%$ of the urban population) were covered by employer-based health insurance. ${ }^{48}$ This study, which aligns research objectives with current health challenges, is embedded in the employer-based health services model, and can thereby foster knowledge dissemination and translation among such employers. Importantly, the rigorous methodological design and health data collection, follow-up into retirement, and linkage of health screening data with insurance claims and medical records, will provide a rich opportunity to investigate disease trajectories and outcomes, and inform approaches to population-based healthcare delivery. Other Chinese population-based studies, such as the China National Diabetes and Metabolic Disorders Study (2007-2008), while nationally representative, are crosssectional and less comprehensive. ${ }^{18} 47$ The Singapore Chinese Health Study ${ }^{49}$ and the Chinese Health and Retirement Longitudinal Study (CHARLS) ${ }^{50}$ provide data on older populations, though they are not focused on CVD and have limited follow-up data. To date, there are no longitudinal, population-based cardiovascular studies of the urban, working population of China. The study is supported by the Fuwai Hospital, NCCD-a clinical research group with longstanding expertise in standardised data abstraction, cataloguing and linkage, ensuring excellent quality control, and organisation for scientific investigation. ${ }^{51-53}$

There are some limitations to this study. The population represents a single company and may not be representative of the larger population of China. Additionally, employed individuals may be healthier than the general population, which may influence the relationship between risk factors and disease onset. However, the study subjects from the Qingdao Port Company are from diverse sectors of the workplace; moreover, the data set is rich with health behaviours and socioeconomic information, which may account for variation in associations within the population. The focus on an employee population is consistent with new models of knowledge discovery and health promotion. ${ }^{54}$ Additionally, longitudinal follow-up of a stable population has the advantage of allowing for insights into risk trajectories and outcomes. Second, participants are recruited from an annual health screening, and so we lack information about employees who are eligible but do not participate in the health screening. However, very few employees decline participation; nonetheless, processes for collecting this information are being developed. Related to this, it is possible that, in some cases, responses to the interview questions might be influenced by the participants' perceptions that their answers might affect their employment status. Third, the collection of outcomes for any longitudinal study is a challenge. The current mechanisms, including self-report and claims data of services rendered through the Qingdao Medical Insurance Bureau are already more advanced than most population-based studies. In the first year of attempting to obtain medical records, over half were successfully obtained. Further efforts are needed to improve this proportion, though this can be accomplished. Additionally, the ascertainment of claims data allows for extensive utilisation and cost analyses, which are unique to this study.

\section{Collaboration}

The Qingdao Port Cardiovascular Study is ongoing, with increasing capacity to adjudicate cardiovascular outcomes, enhancing self-reported outcomes with insurance claims data and hospital medical records. The publications committee and data analytic centre are comprised of researchers from the NCCD, Fuwai Hospital, Beijing, and from Yale University-two institutions with a longstanding partnership and commitment to improving cardiovascular health in China. At this time, all research will be conducted in collaboration with the study investigators. Prospective collaborators are encouraged to contact either JLu (jiapeng.lu@fwoxford.org) or ESS (Erica.spatz@yale.edu).

China's unique position of being a low-income to middle-income country with significant intellectual resources-as well as shared goals between academic, private and public domains-can provide important lessons to the world on the effects of globalisation and the tools needed to protect the health of the public. Longitudinal, population-based studies play an important part in this learning. The Qingdao Port Cardiovascular Health Study is designed to fill these knowledge gaps, and to provide important insights for the public and healthcare system at large.

\section{Author affiliations}

${ }^{1}$ Center for Outcomes Research and Evaluation, Yale University/Yale-New Haven Hospital, New Haven, Connecticut, USA

${ }^{2}$ Section of Cardiovascular Medicine, Yale School of Medicine, New Haven, Connecticut, USA

${ }^{3}$ Section of Cardiovascular Medicine, Qingdao Fuwai Hospital, Qingdao, People's Republic of China

${ }^{4}$ National Clinical Research Center of Cardiovascular Diseases, State Key Laboratory of Cardiovascular Disease, Fuwai Hospital, National Center for Cardiovascular Diseases, Chinese Academy of Medical Sciences and Peking Union Medical College, Beijing, People's Republic of China

${ }^{5}$ Division of Cardiology, University of Colorado Anschutz Medical Campus, Aurora, Colorado, USA

${ }^{6}$ Department of Health Outcomes Research, Saint Luke's Mid America Heart Institute/University of Missouri-Kansas City, Kansas City, Missouri, USA ${ }^{7}$ Qingdao Fuwai Hospital, Qingdao, People's Republic of China

${ }^{8}$ Center for Healthcare Advancement \& Outcomes, Baptist Health South Florida, Miami, Florida, USA

${ }^{9}$ Miami Cardiac \& Vascular Institute, Baptist Health South Florida, Miami, Florida, USA 
${ }^{10}$ The Robert Wood Johnson Foundation Clinical Scholars Program, Department of Internal Medicine, Yale School of Medicine, New Haven, Connecticut, USA

${ }^{11}$ Department of Health Policy and Management, Yale School of Public Health, New Haven, Connecticut, USA

Acknowledgements The authors would like to thank Dr Bingnan Zhang, MD, MBA, for her assistance with the translation of documents and contributions to the manuscript.

Contributors XJ, XLiu, JLi, HMK and LJ led the protocol design; ESS and JLu drafted the manuscript, assembled historical documents of study design and protocols, and reviewed processes for data collection; YW analysed data and provided critical review of the manuscript; FAM, JAS, NSD, KN, HMK and LJ provided critical review of the study design and manuscript.

Funding This study was funded by the Qingdao Port Group and Fuwai Hospital, National Center for Cardiovascular Diseases.

Competing interests None declared.

Ethics approval The ethics committees of the Qingdao Fuwai Hospital and the Fuwai Hospital, NCCD (1 March 2013), and Yale University (11 December 2013), approved the study.

Provenance and peer review Not commissioned; externally peer reviewed.

Data sharing statement No additional data are available.

Open Access This is an Open Access article distributed in accordance with the Creative Commons Attribution Non Commercial (CC BY-NC 4.0) license, which permits others to distribute, remix, adapt, build upon this work noncommercially, and license their derivative works on different terms, provided the original work is properly cited and the use is non-commercial. See: http:// creativecommons.org/licenses/by-nc/4.0/

\section{REFERENCES}

1. Mahmood SS, Levy D, Vasan RS, et al. The Framingham Heart Study and the epidemiology of cardiovascular disease: a historica perspective. Lancet 2014;383:999-1008.

2. No authors listed]. The Atherosclerosis Risk in Communities (ARIC) Study: design and objectives. The ARIC investigators. Am J Epidemiol 1989;129:687-702.

3. Bild DE, Bluemke DA, Burke GL, et al. Multi-ethnic study of atherosclerosis: objectives and design. Am J Epidemiol 2002;156:871-81

4. Keku E, Rosamond W, Taylor HA Jr, et al. Cardiovascular disease event classification in the Jackson Heart Study: methods and procedures. Ethn Dis 2005;15(4 Suppl 6):S6-62-70.

5. Liu J, Hong Y, D'Agostino RB Sr, et al. Predictive value for the Chinese population of the Framingham $\mathrm{CHD}$ risk assessment tool compared with the Chinese Multi-Provincial Cohort Study. JAMA 2004;291:2591-9.

6. Ueshima H, Sekikawa A, Miura K, et al. Cardiovascular disease and risk factors in Asia: a selected review. Circulation 2008;118: 2702-9.

7. Kuulasmaa K, Tunstall-Pedoe H, Dobson A, et al. Estimation of contribution of changes in classic risk factors to trends in coronaryevent rates across the WHO MONICA Project populations. Lancet 2000;355:675-87.

8. Tunstall-Pedoe $\mathrm{H}$. Autres pays, autres moeurs. BMJ 1988;297:1559-60.

9. Evans AE, Ruidavets JB, McCrum EE, et al. Autres pays, autres coeurs? Dietary patterns, risk factors and ischaemic heart disease in Belfast and Toulouse. QJM 1995;88:469-77.

10. Gupta M, Singh N, Verma S. South Asians and cardiovascular risk: what clinicians should know. Circulation 2006;113:e924-9.

11. Sheth T, Nair C, Nargundkar M, et al. Cardiovascular and cancer mortality among Canadians of European, south Asian and Chinese origin from 1979 to 1993: an analysis of 1.2 million deaths. CMAJ 1999;161:132-8.

12. Mariotti S, Capocaccia R, Farchi G, et al. Differences in the incidence rate of coronary heart disease between north and south European cohorts of the Seven Countries Study as partially explained by risk factors. Eur Heart $J$ 1982;3:481-7

13. Liu M, Wang J, Jiang B, et al. Increasing prevalence of metabolic syndrome in a Chinese elderly population: 2001-2010. PLOS ONE 2013;8:e66233
14. Chen Z, Peto R, Collins R, et al. Serum cholesterol concentration and coronary heart disease in population with low cholesterol concentrations. BMJ 1991;303:276-82.

15. Lawes CM, Rodgers A, Bennett DA, et al. Blood pressure and cardiovascular disease in the Asia Pacific region. $J$ Hypertens 2003;21:707-16.

16. Zhang LF, Yang J, Hong Z, et al. Proportion of different subtypes of stroke in China. Stroke 2003;34:2091-6.

17. Hu SS, Kong LZ, Gao RL, et al. Outline of the report on cardiovascular disease in China, 2010. Biomed Environ Sci 2012;25:251-6.

18. Yang ZJ, Liu J, Ge JP, et al. Prevalence of cardiovascular disease risk factor in the Chinese population: the 2007-2008 China National Diabetes and Metabolic Disorders Study. Eur Heart $J$ 2012;33:213-20.

19. Modesti PA, Agostoni P, Agyemang C, et al. ESH Working Group on Hypertension and Cardiovascular Risk in Low Resource Settings. Cardiovascular risk assessment in low-resource settings: a consensus document of the European Society of Hypertension Working Group on Hypertension and Cardiovascular Risk in Low Resource Settings. J Hypertens 2014;32:951-60.

20. Yang G, Wang $Y$, Zeng $Y$, et al. Rapid health transition in China, 1990-2010: findings from the Global Burden of Disease Study 2010. Lancet 2013;381:1987-2015.

21. Zheng Y, Stein R, Kwan T, et al. Evolving cardiovascular disease prevalence, mortality, risk factors, and the metabolic syndrome in China. Clin Cardiol 2009;32:491-7.

22. World Health Organization Western Pacific Region: Cardiovascular Disease in China. Secondary World Health Organization Western Pacific Region: Cardiovascular Disease in China, 2014. http://www wpro.who.int/china/mediacentre/factsheets/cvd/en/

23. $\mathrm{Xu} \mathrm{Y}$, Wang $\mathrm{L}, \mathrm{He} \mathrm{J}$, et al. Prevalence and control of diabetes in Chinese adults. JAMA 2013;310:948-59.

24. Yusuf S, Reddy S, Ounpuu S, et al. Global burden of cardiovascular diseases: part I: general considerations, the epidemiologic transition, risk factors, and impact of urbanization. Circulation 2001;104:2746-53.

25. Smith S, Ralston J, Taubert K. Urbanization and cardiovascular disease: raising heart-healthy children in today's cities. Geneva: The World Heart Federation, 2012.

26. Sun $Q$, Hong X, Wold LE. Cardiovascular effects of ambient particulate air pollution exposure. Circulation 2010;121:2755-65.

27. Xie W, Li G, Zhao D, et al. Relationship between fine particulate air pollution and ischaemic heart disease morbidity and mortality. Heart 2015;101:257-63.

28. Brook RD, Rajagopalan S, Pope CA III, et al. Particulate matter air pollution and cardiovascular disease: an update to the scientific statement from the American Heart Association. Circulation 2010;121:2331-78.

29. Pan A, Malik VS, Hu FB. Exporting diabetes mellitus to Asia: the impact of Western-style fast food. Circulation 2012;126: 163-5.

30. Popkin BM. Synthesis and implications: China's nutrition transition in the context of changes across other low-income and middle-income countries. Obes Rev 2014;15(Suppl 1):60-7.

31. He J, Gu D, Chen J, et al. Premature deaths attributable to blood pressure in China: a prospective cohort study. Lancet 2009;374:1765-72.

32. Odegaard AO, Koh WP, Yuan JM, et al. Western-style fast food intake and cardiometabolic risk in an eastern country. Circulation 2012;126:182-8.

33. He J, Gu D, Wu X, et al. Major causes of death among men and women in China. N Engl J Med 2005;353:1124-34.

34. Zhou T, Li X, Tang Z, et al. Risk factors of CVD mortality among the elderly in Beijing, 1992-2009: an 18-year cohort study. Int J Environ Res Public Health 2014;11:2193-208.

35. Qian Z, He Q, Lin HM, et al, HEI Health Review Committee. Part 2. Association of daily mortality with ambient air pollution, and effect modification by extremely high temperature in Wuhan, China. Res Rep Health Eff Inst 2010;154:91-217.

36. Wu Z, Yao C, Zhao D, et al. Sino-MONICA project: a collaborative study on trends and determinants in cardiovascular diseases in China, part i: morbidity and mortality monitoring. Circulation 2001;103:462-8.

37. No authors listed]. The World Health Organization MONICA Project (monitoring trends and determinants in cardiovascular disease): a major international collaboration. WHO MONICA Project Principal Investigators. J Clin Epidemiol 1988;41:105-14.

38. Zhang W, Sun K, Yang Y, et al. Plasma uric acid and hypertension in a Chinese community: prospective study and metaanalysis. Clin Chem 2009;55:2026-34. 
39. Shang X, Li J, Tao Q, et al. Educational level, obesity and incidence of diabetes among Chinese adult men and women aged 18-59 years old: an 11-year follow-up study. PLOS ONE 2013;8: e66479.

40. Chen Z, Chen J, Collins R, et al. China Kadoorie Biobank of 0.5 million people: survey methods, baseline characteristics and long-term follow-up. Int J Epidemiol 2011;40:1652-66.

41. Li Q, Babor TF, Hao W, et al. The Chinese translations of Alcohol Use Disorders Identification Test (AUDIT) in China: a systematic review. Alcohol Alcohol 2011;46:416-23.

42. Macfarlane DJ, Lee CC, Ho EY, et al. Reliability and validity of the Chinese version of IPAQ (short, last 7 days). J Sci Med Sport 2007;10:45-51.

43. Duale N, Lipkin WI, Briese T, et al. Long-term storage of blood RNA collected in RNA stabilizing Tempus tubes in a large biobankevaluation of RNA quality and stability. BMC Res Notes 2014;7:633.

44. Li J, Li X, Wang Q, et al China PEACE Collaborative Group. ST-segment elevation myocardial infarction in China from 2001 to 2011 (the China PEACE-Retrospective Acute Myocardial Infarction Study): a retrospective analysis of hospital data. Lancet 2015;385:441-51.

45. Sun S, Chen J, Johannesson $M$, et al. Population health status in China: EQ-5D results, by age, sex and socio-economic status, from the National Health Services Survey 2008. Qual Life Res 2011;20:309-20.

46. Gu D, Gupta A, Muntner P, et al. Prevalence of cardiovascular disease risk factor clustering among the adult population of China: results from the International Collaborative Study of Cardiovascular Disease in Asia (InterAsia). Circulation 2005;112:658-65.

47. Huang Y, Gao L, Xie X, et al. Epidemiology of dyslipidemia in Chinese adults: meta-analysis of prevalence, awareness, treatment, and control. Popul Health Metr 2014;12:28.

48. Hu S, Tang S, Liu Y, et al. Reform of how health care is paid for in China: challenges and opportunities. Lancet 2008;372:1846-53.

49. Hankin JH, Stram DO, Arakawa K, et al. Singapore Chinese Health Study: development, validation, and calibration of the quantitative food frequency questionnaire. Nutr Cancer 2001;39:187-95.

50. Strauss J, Lei X, Park A, et al. Health outcomes and socio-economic status among the elderly in Gansu and Zhejiang Provinces, China: evidence from the CHARLS Pilot. J Popul Ageing 2010;3:111-42.

51. Li J, Dharmarajan K, Li X, et al. Protocol for the China PEACE (Patient-centered Evaluative Assessment of Cardiac Events) retrospective study of coronary catheterisation and percutaneous coronary intervention. BMJ Open 2014;4:e004595.

52. Chen ZM, Pan HC, Chen YP, et al. Early intravenous then oral metoprolol in 45,852 patients with acute myocardial infarction: randomised placebo-controlled trial. Lancet 2005;366:1622-32.

53. Chen ZM, Jiang LX, Chen YP, et al. Addition of clopidogrel to aspirin in 45,852 patients with acute myocardial infarction: randomised placebo-controlled trial. Lancet 2005;366:1607-21.

54. Ogunmoroti O, Younus A, Rouseff M, et al. Assessment of American Heart Association's Ideal Cardiovascular Health Metrics Among Employees of a Large Healthcare Organization: the Baptist Health South Florida Employee Study. Clin Cardiol 2015;38:422-9. 